\title{
Transverse instabilities in chemical Turing patterns of stripes
}

\author{
B. Peña, ${ }^{1}$ C. Pérez-García, ${ }^{1, *}$ A. Sanz-Anchelergues, ${ }^{2}$ D. G. Míguez, ${ }^{2}$ and A. P. Munuzuri ${ }^{2}$ \\ ${ }^{1}$ Instituto de Física, Universidad de Navarra, E-31080 Pamplona, Spain \\ ${ }^{2}$ Grupo de Física No lineal, Universidad Santiago de Compostela, E-15782 Santiago, Spain
}

(Received 23 June 2003; published 18 November 2003)

\begin{abstract}
We present a theoretical and experimental study of the sideband instabilities in Turing patterns of stripes. We compare numerical computations of the Brusselator model with experiments in a chlorine dioxide-iodinemalonic acid (CDIMA) reaction in a thin gel layer reactor in contact with a continuously refreshed reservoir of reagents. Spontaneously evolving Turing structures in both systems typically exhibit many defects that break the symmetry of the pattern. Therefore, the study of sideband instabilities requires a method of forcing perfect, spatially periodic Turing patterns with the desired wave number. This is easily achieved in numerical simulations. In experiments, the photosensitivity of the CDIMA reaction permits control and modulation of Turing structures by periodic spatial illumination with a wave number outside the stability region. When a too big wave number is imposed on the pattern, the Eckhaus instability may arise, while for too small wave numbers an instability sets in forming zigzags. By means of the amplitude equation formalism we show that, close to the hexagon-stripe transitions, these sideband instabilities may be preceded by an amplitude instability that grows transient spots locally before reconnecting with stripes. This prediction is tested in both the reaction-diffusion model and the experiment.
\end{abstract}

DOI: 10.1103/PhysRevE.68.056206

PACS number(s): 82.40.Ck, 82.40.Bj, 05.45.-a

\section{INTRODUCTION}

Half a century ago, Turing [1] developed a theory of morphogenesis which has had a profound impact on theoretical developments in pattern formation. Turing showed that stationary concentration patterns may spontaneously develop in an open system containing two reacting substances provided one of them diffuses much faster than the other. Nowadays, the Turing mechanism is still considered a prototype for the formation of coherent patterns in nonequilibrium systems. Despite considerable efforts to verify Turing's proposal experimentally and to find stationary spatial patterns in a real chemical system, it took almost 40 years before the first experimental evidence of convection-free Turing patterns was reported. The disparity in diffusion coefficients assumed in the Turing mechanism was hard to achieve because small molecules in aqueous solution have diffusion coefficients that do not differ substantially from each other.

Castets et al., working with an open, continuously fed unstirred reactor (CFUR) observed spatial pattern formation arising from a homogeneous steady state in the chloriteiodide-malonic acid (CIMA) reaction [2]. Since then, Turing patterns have been extensively studied in the CIMA reaction and in its variant, the chlorine dioxide-iodine-malonic acid (CDIMA) reaction [3]. In these experiments, sufficient differences in the mobilities were achieved by using a macromolecular indicator that partially immobilizes the "critical" species by reversible complexations. Depending on the control parameters (concentration of reactants and diffusion coefficients), the dynamics of this reaction exhibits several kinds of steady spatially periodic patterns close to onset: hexagons, stripes, and "rhombs" [4]. (Usually the so-called black eyes arise as secondary modes far from threshold [4].)

\footnotetext{
*Electronic address: carlos@fisica.unav.es
}

Turing-like concentration patterns have also been observed during the irreversible polymerization of acrylamide in an oxygen atmosphere in the presence of methylene blue sulfide [5]. But the main drawbacks of this system are that, once the polymerization is over, the pattern cannot be changed by further external perturbation, and that the driving instability mechanism is still under discussion [6-8]. In a recent theoretical work [9], it was suggested that a certain class of electrochemical systems might exhibit Turing-type structures without suffering from the restriction on the different rates of the transport processes. This therefore opens promising perspectives in the study of further Turing-like structures.

The stability of patterns against spatial modulations is a key issue, because long-wave instabilities are pattern selection mechanisms in systems with translation symmetry. In the case of a pattern of rolls, the Eckhaus or the zigzag instability may appear when the homogeneous translational invariance is spontaneously broken. Spatial modulations of patterns have been much studied in convective fluids [10,11], but, to our knowledge, they have scarcely been discussed in chemistry. On the theoretical side, the three instabilities of striped patterns (cross roll, Eckhaus, and zigzag) were well reproduced within the chemical Schnackenberg model [12]. Experimentally, illumination and electric fields have been used to modify Turing-like patterns obtained during polymerization in the acrylamide-methylene blue-sulfide-oxygen reaction, and the same system has been exposed to spatially periodic light perturbation [6]. Recently, Muñuzuri et al. [13] have revealed the sensitivity of the CDIMA reaction to visible light and proposed a simple model for its photosensitivity. This study opened the possibility of controlling Turing patterns by illumination.

Our main aim in the present work is to discuss the mechanism of modulational instabilities in chemical systems, by comparing numerical simulations of the Brusselator model 
with experiments in a CFUR reactor with the CDIMA reaction. In Sec. II we summarize the stability limits obtained previously for the different patterns in the model. In Sec. III, the experimental setup is introduced. Both the model and the experiments display hexagons and stripes as stationary solutions, but we focus our attention on patterns of stripes. The stability of stripes, in terms of amplitude equations [14], is discussed in Sec. IV. Close to the hexagon-stripe transition, unstable hexagons may alter the modulational destabilizing mechanisms of stripes. In Sec. V, we analyze the Eckhaus instability, and in Sec. VI the zigzag instability in these systems is discussed. The paper ends with conclusions and a discussion of the results in Sec. VII.

\section{STATIONARY PATTERNS IN THE BRUSSELATOR MODEL}

We have chosen the Brusselator model because it permits analytical calculations in qualitative agreement with experiments [15]. It consists of two coupled reaction-diffusion equations:

$$
\begin{gathered}
\partial_{t} X=A-(B+1) X+X^{2} Y+\nabla^{2} X, \\
\partial_{t} Y=B X-X^{2} Y+D \nabla^{2} Y,
\end{gathered}
$$

where $X$ and $Y$ stand for the concentrations of activator and substrate, respectively, $D$ is the diffusion ratio of the two species, and $A$ and $B$ are constant parameters, one of them $(B)$ being selected as the control parameter. For $\eta \equiv \sqrt{1 / D}$ $<\left(\sqrt{1+A^{2}}-1\right) / A$, the homogeneous steady solution $\left(X_{s}, Y_{s}\right)=(A, B / A)$ becomes unstable against stationary perturbations, leading to a Turing pattern. The threshold value for the Turing instability is $B_{c}=(1+A \eta)^{2}$, with a critical wave number $k_{c}=\sqrt{A \eta}$. From now on, it is convenient to deal with the rescaled control parameter $\mu=\left(B-B_{c}\right) / B_{c}$, known as supercriticality.

Results on the spatial stability of hexagonal Turing patterns in this model have been extensively reported in previous work $[16,17]$. Special attention has been paid to the transition between hexagons and stripes. The main results are summarized in Fig. 1. Hexagons are stable inside the shaded regions. More specifically, hexagons with a total phase summing to $\pi$, denoted as $H_{\pi}$ hexagons, are stabilized at threshold (bottom of Fig. 1). These coexist with stripes for $\mu$ $<\mu_{H}$. Stripes are the sole stable solution within the area with oblique lines. Thereafter, hexagons with a zero total phase $\left(H_{0}\right.$, or reentrant hexagons [18]) coexist with stripes. For $\mu>\mu_{S}$ only $H_{0}$ hexagons remain stable. These boundaries were obtained by using the amplitude equation formalism, which is valid only when sufficiently close to threshold. Another interesting result previously described $[16,17]$ is that the average wave number in simulations increases when $\mu$ increases, a relevant fact in the wave number selection process.

To check the validity of these stability regions, we perform numerical integrations of the Brusselator model with fixed values $A=4.5$ and $A \eta=1.59$. A simple but efficient odd-even hopscotch method [12] on a mesh of $128 \times 128$

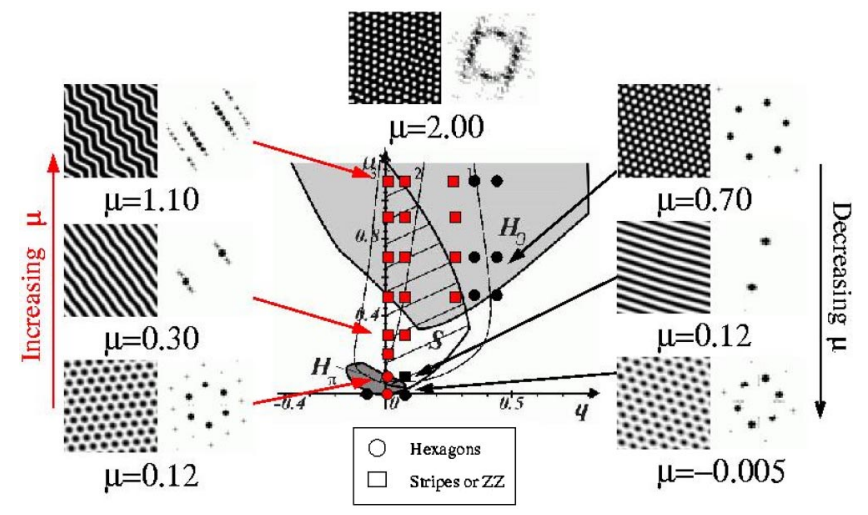

FIG. 1. Stability regions for hexagons (shaded regions) and stripes (striped area) in the Brusselator model obtained from the amplitude equations [Eq. (2) (see Ref. [17])]. Simulations and Fourier transforms for different values of the supercriticality $\mu$ and excess wave number $q_{i}=k_{i}-k_{c}$ are shown.

points with periodic boundary conditions is used. The concentration $X$ is represented in a gray scale varying from black (minimum) to white (maximum) in Fig. 1. In the same figure we also gather the patterns resulting from integration and their corresponding Fourier transforms for several values of $\mu$.

We begin with a random initial mesh and let the model evolve until a stationary state is reached. Then, the supercriticality $\mu$ is increased in a small step $\Delta \mu \simeq 0.02$ and the resulting pattern is recorded. Near threshold $(\mu=0.12)$, an initial random mesh evolves into a perfect pattern of $H_{\pi}$ hexagons. When the upper stability limit for $H_{\pi}$ hexagons is crossed $(\mu=0.30)$, these solutions are replaced by stripes with the same wave number as the hexagons. Since this wave number is too small, the stripes become wavy and their Fourier transforms exhibit a pair of off-axis satellite modes. Further increase in $\mu$ gives rise to zigzags $(\mu=1.10)$, which display five main pairs of modes, the critical one and four off critical. These modes have the same horizontal wave number component and they become more intense when $\mu$ is raised. The angle between zigs and zags decreases when $\mu$ increases, approaching $2 \pi / 3 \mathrm{rad}$. When the stability region of $H_{0}$ hexagons is entered $(\mu=2.00)$, the off-axis modes become the most intense. Finally, zigzags end up in very distorted $H_{0}$ hexagons with a higher wave number.

In a second run this hexagonal pattern $(\mu=2)$ is taken as the initial condition, but $\mu$ is diminished. After the first decrease the pattern becomes more ordered $(\mu=0.70)$, but it is made up of slightly distorted hexagons. Its Fourier transform shows three peaks with different values. This pattern remains unchanged on lowering $\mu$ until, for $\mu \leqslant 0.4$, the stripes become stable and replace the hexagons. The remaining mode has a too long wave number, becomes unstable, and is replaced by a single mode with a different orientation and a lower wave number. For even smaller $\mu, H_{\pi}$ hexagons replace stripes.

These simulations show that, starting from suitable initial conditions, wave number and planform selection may be 


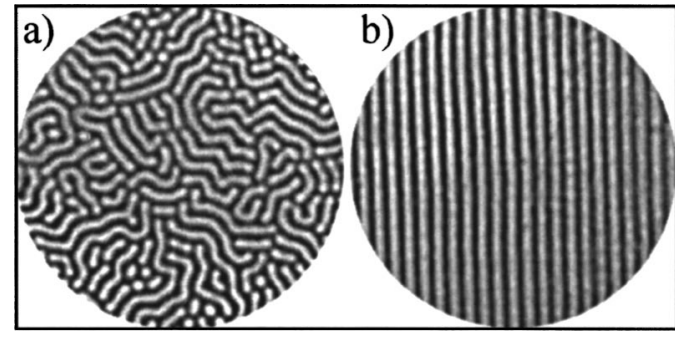

FIG. 2. Patterns of stripes in experiments for the same concentration values. (a) Spontaneous pattern. (b) Spatially forced pattern.

studied. In the following sections we focus our attention on the sideband instabilities (Eckhaus and zigzag instabilities) in patterns of stripes.

\section{EXPERIMENTAL SETUP}

Experiments on the CDIMA reaction have been performed in a thermostated CFUR (see Refs. [19], [20]) at $4 \pm 0.5^{\circ} \mathrm{C}$. It consists of a continuously fed stirred tank reactor (CSTR) where the reagents are mixed and in contact with a thin agarose gel layer (2\% agarose, $0.3 \mathrm{~mm}$ thickness, 20 $\mathrm{mm}$ diameter). The gel layer is separated from the feeding tank by two membranes: a nitrocellulose membrane (Schleicher \& Schnell, pore size $0.45 \mathrm{~mm}$ ) and an Anapore membrane impregnated with $0.5 \%$ agarose gel (Whatman, pore size $0.2 \mu \mathrm{m})$. In order to ensure that the arising structure is two dimensional, we select a thickness of the gel smaller than the wavelength exhibited by the system for the concentrations selected in this experiments. The reagents were fed into the CSTR by a peristaltic pump, previously calibrated to ensure the correct control in the concentration values. The input concentration of reagents were as follows: variable $\left[\mathrm{ClO}_{2}\right]=0.07$ and $=0.09 \mathrm{mM}$, and fixed $\left[\mathrm{I}_{2}\right]=0.45 \mathrm{mM}$, $\left[\mathrm{H}_{2} \mathrm{SO}_{4}\right]=10 \mathrm{mM}$, and [malonic acid] $=1.2 \mathrm{mM}$. We added polyvinyl alcohol (PVA) at $[\mathrm{PVA}]=10 \mathrm{~g} / \mathrm{l}$, as an indicator of the activator concentration and to increase the ratio between the diffusion coefficients of activator and inhibitor. In this way, parts with high activator concentration exhibit garnet coloration, while zones in which the inhibitor is dominant show light yellow color. For $\left[\mathrm{ClO}_{2}\right]=0.07 \mathrm{mM}$, spontaneous stable stripes are formed with a wavelength $\lambda_{c}=0.54$ $\pm 0.02 \mathrm{~mm}$, while for the second value, $\left[\mathrm{ClO}_{2}\right]=0.09 \mathrm{mM}$, the steady pattern of stripes displays $\lambda_{c}=0.48 \pm 0.02 \mathrm{~mm}$.

The photosensitivity of the CDIMA reaction [13] is used to impose initial patterns on the system [21] by means of illumination from the side with a $150 \mathrm{~W}$ halogen lamp. Before reaching the reactive gel, the light of the lamp is filtered through a slide with a static pattern of parallel black and transparent bands. Varying the width of these bands, different wavelengths may be forced in the pattern. An example of spontaneous and initially forced patterns of stripes is shown in Fig. 2. The periodically perturbed pattern [Fig. 2(b)] has the same wave number as the spontaneous pattern [Fig. 2(a)] and remains stationary in time.

Standard protocol for the experiments always begins by pumping reagents. After $4 \mathrm{~h}$, stationary stripes cover the whole media. Then the pattern is deleted by homogeneous light intensity $(150 \mathrm{~W})$ applied for $20 \mathrm{~min}$, after which we shone the desired perturbation through the slide for $10 \mathrm{~min}$. Afterward, the slide is removed $(t=0)$ and the unstable pattern begins to evolve.

The experimental control parameter is the concentration of chlorine dioxide $\left[\mathrm{ClO}_{2}\right]$. We select two values which give stable patterns of stripes. In one case the stripes are close to the stable range of hexagons and in the second case we remain close to the border of stability of hexagonal Turing structures. For different imposed wave numbers, the pattern is unstable and, once the transparency is removed, it starts to evolve into a more stable situation.

\section{AMPLITUDE EQUATIONS}

A weakly nonlinear analysis around the stationary reference state gives the so-called amplitude equations which for the three modes that form hexagons take the following form:

$$
\begin{aligned}
\tau_{0} \partial_{t} A_{1}= & \mu A_{1}+\partial_{x_{1}}^{2} A_{1}+v \bar{A}_{2} \bar{A}_{3}-\left|A_{1}\right|^{2} A_{1} \\
& -h\left(\left|A_{2}\right|^{2}+\left|A_{3}\right|^{2}\right) A_{1},
\end{aligned}
$$

where the subscripts in the derivatives stand for $\partial_{x_{i}}=\hat{n}_{i} \cdot \nabla$, and $\hat{n}_{i}$ denote unit vectors in the direction of the three hexagonal modes. Companion equations for $A_{2}$ and $A_{3}$ are simply obtained by subscript permutations.

Beyond a threshold value hexagons are replaced by stripes. Without loss of generality we assume that stripes select the mode $\hat{n}_{1}=(1,0)$, and Eq. (2) must be replaced by the Newell-Whitehead-Segel (NWS) equation [14]

$$
\partial_{t} A_{r}=\mu A_{r}+\left(\partial_{x}-\frac{i}{2 k_{c}} \partial_{y}^{2}\right)^{2} A_{r}-\left|A_{r}\right|^{2} A_{r}
$$

in which the spatial term accounts now for the different scaling in the $x$ and $y$ directions [14].

\section{A. Sideband instabilities in striped patterns}

The last equation has a stationary solution $A_{r}$ $=\sqrt{\mu-q^{2}} e^{i q x}\left(\mu>q^{2}\right)$. Perturbations around this solution take the form $A_{r}=\left(\sqrt{\mu-q^{2}}+r\right) e^{i q x+\phi(x, y, t)}$. A linear stability analysis gives at lowest order the well-known phase equation [14]

$$
\partial_{t} \phi=D_{\|} \partial_{x}^{2} \phi+D_{\perp} \partial_{y}^{2} \phi
$$

with $D_{\|}=\left(\mu-3 q^{2}\right) /\left(\mu-q^{2}\right)$ and $D_{\perp}=q / k_{c}$. Therefore, for $q^{2}>q_{E}^{2}=\mu / 3$ the Eckhaus instability arises, while for $q<0$ modulations perpendicular to the stripe axis can grow, producing a zigzag instability. Although these limits fail to describe sideband instabilities far from threshold appropriately, we shall keep these expressions as a reference in the following discussions.

The scenario can be different near the hexagon-stripe transition. When hexagons are slightly unstable, a lowering in the amplitude of stripes should lead, either globally or locally, to a transverse amplitude instability. Although small, 
the quadratic resonant term in Eq. (2) may induce hexagons in places where the phase gradient increases.

To discuss amplitude stabilization of transverse modes, let us consider the stripe solution in Eq. (2), $A_{1}=A_{r}=\mathcal{A} e^{i q_{A} x}$, $A_{2}=A_{3}=0$, where $q_{A}=k_{A}-k_{c}$. Bands with $|\mathcal{A}|^{2}=\mu-q_{A}^{2}$ are stationary solutions of Eq. (2), provided $h>1$. They could be unstable not only through phase modes, as assumed in Eq. (4), but also for transversal amplitude perturbations. The quadratic term should favor resonant triads of modes satisfying the conditions $\vec{k}_{A}+\vec{k}_{B}+\vec{k}_{C}=\overrightarrow{0} \quad\left(\vec{q}_{A}+\vec{q}_{B}+\vec{q}_{C}\right.$ $=\overrightarrow{0}$ ). For simplicity, we shall consider only the particular case of squeezed hexagonal perturbations that obey the conditions $q_{A x}=-(1 / 2) q_{B x}=-(1 / 2) q_{C x}, \quad q_{B y}=-q_{C y}$. (The general case of sheared hexagons is much more difficult to deal with analytically.) Therefore, we assume that perturbations in the form $A_{1}=A=(\mathcal{A}+a) e^{i q_{A} x},\left|A_{2}\right|^{2}=\left|A_{3}\right|^{2}=b A_{2}$ $=b e^{i q_{B}\left(\hat{n}_{B} \cdot \vec{r}\right)}$ act on the system. Here $\hat{n}_{B}$ indicates the unit vector along a transverse mode $B$. After linearizing we obtain two uncoupled equations:

$$
\begin{gathered}
\partial_{t} a=\left(\mu-q_{A}^{2}\right) a-3 \mathcal{A}^{2} a, \\
\partial_{t} b=\left(\mu-q_{B}^{2}\right) b-h \mathcal{A}^{2} a+v \mathcal{A} b .
\end{gathered}
$$

The first equation does not depend on $b$. As $v \mathcal{A} \ll h|\mathcal{A}|^{2}$ (slight subcriticality) Eq. (5b) can be written in the form

$$
\partial_{t} b=\mu-q_{B}^{2}-h\left(\mu+q_{A}^{2}\right),
$$

so, as the right-hand side becomes positive, transverse amplitude modes may destabilize a pattern of stripes. Notice that $q_{B}^{2} \geqslant(1 / 4) q_{A}^{2}$, so that stationary stripes can become unstable whenever

$$
q_{A}^{2} \geqslant \frac{h-1}{h-1 / 4} \mu
$$

Whether stripes will suffer a short-wave-number or an amplitude instability leading to transient distorted hexagons depends on the details of the reactive system. For each particular system, the stability threshold in Eq. (7) has to be compared with the corresponding sideband stability limit.

\section{ECKHAUS INSTABILITY}

Detailed theoretical and numerical analysis of this instability under the NWS equation framework can be found in Ref. [22]. In general, this instability is difficult to observe experimentally owing to the appearance of other secondary instabilities, the limitation of experimental techniques to induce initial patterns, and the unavoidable effects of lateral boundaries. However, this instability has been reported in rather different physical systems [11].

To describe the Eckhaus instability appropriately in the framework of the NWS equation (3), one must replace the simple linear phase Eq. (4) by an equation in the form [14]

$$
\partial_{t} \phi=D_{\|} \partial_{x}^{2} \phi-D_{4} \partial_{x}^{4} \phi+g \partial_{x}\left[\left(\partial_{x} \phi\right)^{2}\right] .
$$
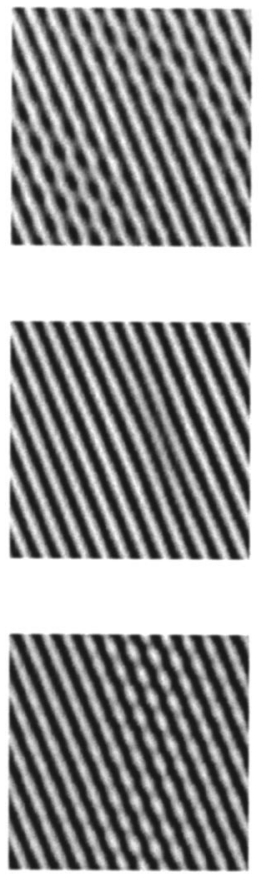

(c)

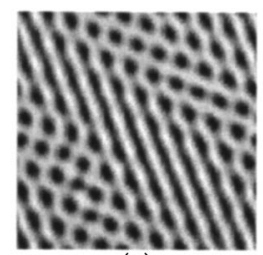

(a)
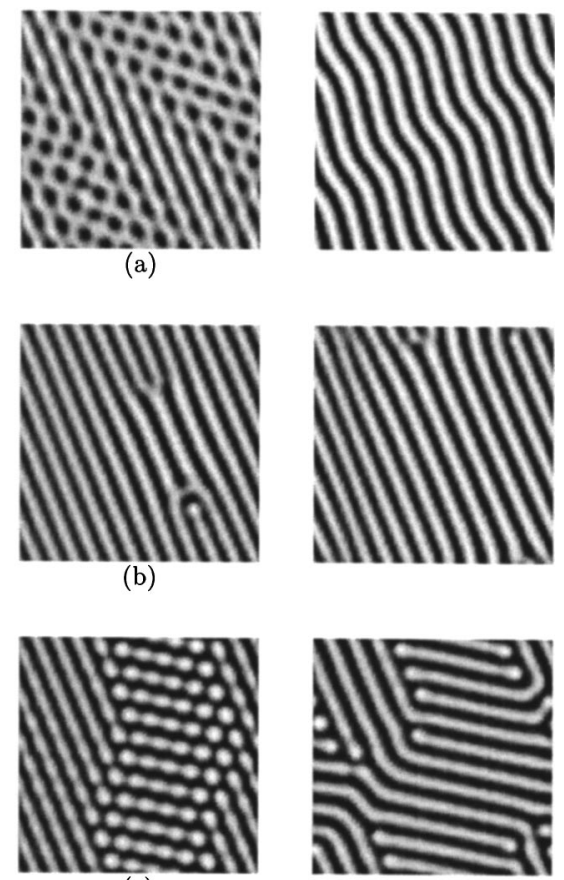

(b)
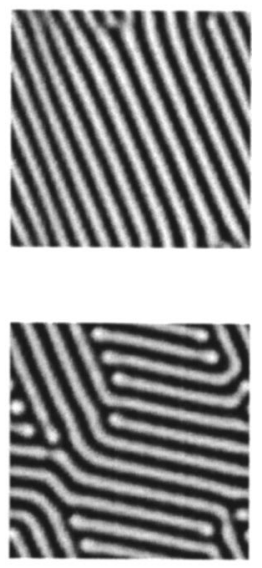

FIG. 3. Numerical simulations of the Brusselator for (a) $\mu=0.09, q=0.26$; (b) $\mu=0.15, q=0.32$; (c) $\mu=0.35, q=0.42$.

In Ref. [22] it was proved that the coefficients $D_{4}$ and $g$ are negative, so that the modulational instability does not saturate and the phase description breaks down. Numerical simulations of NWS equation (3) show that the phase gradients (which give the local wave number) become steeper at some places and the amplitude $A$ is depressed at locations where phase gradients grow. In the case of pure stripes, $A$ could decrease to zero at these places and the phase becomes undefined, leading to the so-called phase slips [22]. In twodimensional systems, this results in nucleation or annihilation of a pair of stripes through dislocations, likely due to the excitation of transverse modes [23].

Comparison of the stability threshold in Eq. (7) with the Eckhaus value $q_{E}^{2}=\mu / 3$ gives the condition $1 \leqslant h \leqslant 11 / 8$. When $h$ lies in this interval the transverse amplitude instability will appear before the Eckhaus instability takes place. For the parameter values taken in the simulations of the Brusselator model, however, we obtain $h=2.9$ and hence a transverse mode can grow only locally, but not globally, at places where $A$ is sufficiently small.

\section{A. Numerical simulations of the Eckhaus instability}

To confirm that such a situation can occur in the Brusselator, we performed numerical computations starting from a pattern of perfect parallel stripes as initial condition, but with their wave number modified by a positive amount $q\left(k=k_{c}\right.$ $+q$ ). This is achieved by changing the spatial step $\Delta x$ in the direction perpendicular to rolls. Three results for stripes with $q$ initially outside the Eckhaus stable region are gathered in Fig. 3. In the first stages, a modulational instability that compresses stripes at some places of the computation lattice is discernable. The amplitude and the wave number diminish at 

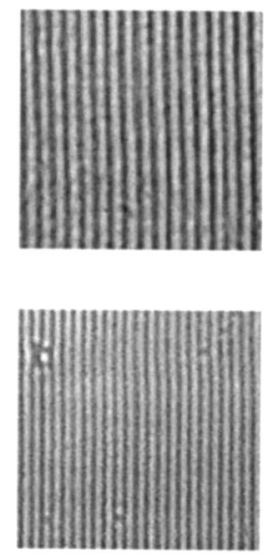

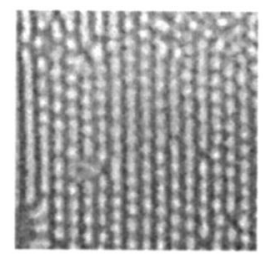

(a)

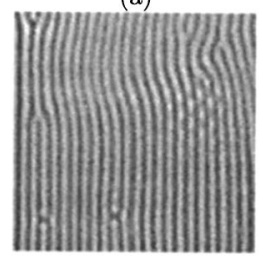

(b)
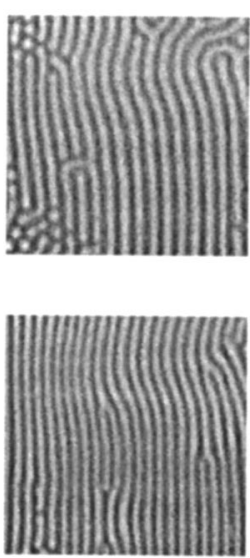

FIG. 4. Experimental Eckhaus instability in CDIMA reaction for two concentrations of chlorine dioxide. (a) $\left[\mathrm{ClO}_{2}\right]=0.07 \mathrm{mM}: t$ $=0,15$, and $60 \mathrm{~min}$. (b) $\left[\mathrm{ClO}_{2}\right]=0.09 \mathrm{mM}: t=0,60$, and $140 \mathrm{~min}$. Both sequences show the temporal evolution (from left to right) of stripes with an initial wave number larger than $\lambda_{c}$.

these locations until some transverse mode grows up locally. In the sequences of Figs. 3(a) and 3(c) the value of $\mu$ is close to the hexagon-stripe transition, so that distorted hexagons are locally made out, but after tilting the stripes and inducing a wave number adjustment they disappear. (Eventually some isolated spots remain trapped inside stripes.) Notice that the mechanism is similar for either $H_{\pi}$ or $H_{0}$ hexagons. For $\mu$ far from the bistability region [Fig. 3(b)] instead, the usual phase slip leads to a pair of dislocations, which climb to the sidewalls [24].

\section{B. Experimental Eckhaus instability}

Concentrations used in the experiments are close to $\left(\left[\mathrm{ClO}_{2}\right]=0.07 \mathrm{mM}\right)$ and far from $\left(\left[\mathrm{ClO}_{2}\right]=0.09 \mathrm{mM}\right)$ the hexagon-stripe transition value. The evolution for the first concentration is shown in Fig. 4(a). We force stripes with an unstable wavelength $\lambda=0.41 \mathrm{~mm}<\lambda_{c}=0.54 \mathrm{~mm}$. During the first stages, the parallel bands break quickly (in just 15 min) into spots. They form transient, quite distorted stretched hexagons. After $60 \mathrm{~min}$, these unstable spots disappear almost completely, the stripes are reoriented [Fig. 4(c)], and their wave number again reaches the stable band.

We repeat the procedure for the second concentration chosen $\left(\left[\mathrm{ClO}_{2}\right]=0.09 \mathrm{mM}\right)$. The evolution of the imposed stripes is shown in Fig. 4(b). Initially, the pattern displays few defects and inhomogeneities at some places. The bigger defect gives rise to a couple of dislocations propagating through the pattern in opposite directions, as one can see from the central snapshot in Fig. 4(b), taken after $60 \mathrm{~min}$. In the same figure the formation of a deformed hexagonal arrangement of transient spots is noticeable, which finally disappears. After $140 \mathrm{~min}$ [to the left in Fig. 4(b)], the pattern exhibits stripes with some dislocations.

\section{THE ZIGZAG INSTABILITY}

For too small wave numbers the translational invariance of stripes is broken by the zigzag instability [11]. Stripes become wavy and domains of tilted stripes in two preferred directions arise in the pattern. This tilting increases the wave number of stripes locally [14].

In the framework of the NWS equation (3), this instability occurs for negative off-critical $(q<0)$ wave numbers. At the lowest order, the linear evolution of zigzags is well described by Eq. (4), but higher order derivatives and nonlinear terms must be added to saturate this instability. By symmetry arguments the following phase equation is deduced [14]:

$$
\partial_{t} \phi=\left[\frac{q}{k_{c}}+\frac{3}{2 k_{c}}\left(\partial_{y} \phi\right)^{2}\right] \partial_{y}^{2} \phi-\frac{1}{4 k_{c}} \partial_{y}^{4} \phi .
$$

After applying $\partial_{y}$ to this equation, taking $\psi=\partial_{y} \phi$ and rescaling time, one arrives at the well-known Cahn-Hilliard equation describing the dynamics of phase separation in conservative systems [25], which is written in the form

$$
\partial_{t} \psi=\partial_{y}^{2} \frac{\delta \mathcal{F}}{\delta \psi}, \quad \mathcal{F} \psi \psi=\int q \frac{\psi^{2}}{2}+\frac{\psi^{4}}{8}+\frac{1}{2}\left(\partial_{y} \psi\right)^{2} d y,
$$

in which $\mathcal{F}$ is the Cahn-Hilliard free energy. (The conserved quantity in our case is the total phase $\Phi=\int_{0}^{\infty} \psi d y$.) This expression admits two kinds of solutions: periodic waves, always unstable [26], and kinks connecting the states $\psi=$ $\pm \sqrt{2 q} y$. A solution with a given periodicity evolves toward another with longer wavelength. Isolated kinks are stable, but an array of kinks undergoes coarsening by an annihilation process and without characteristic length scale. Thus, the average size of the domain enclosed between kinks increases regularly in time. The interaction between two kinks is exponentially decaying, and so weak that tiny external interactions can lock these otherwise unstable configurations [27].

Busse and Auer [28] considered the stability of undulating rolls resulting from a zigzag instability in the NWS amplitude framework. Undulating rolls are unstable against longitudinal modulations for $q_{E}^{2}>\mu / 3$ (Eckhaus limit) and against transverse modes for $q^{2}<\mu / 7$, so that no stable band for undulating rolls exists. In numerical computations, however, undulating rolls are obtained (see Fig. 1). These might be stabilized through some pinning effect due to the finite computation size. As suggested in Ref. [27] the pattern selects few quasiresonant modes, again likely owing to the finiteness of the computation grid or to wave packet effects.

\section{A. Numerical simulations of the zigzag instability}

As discussed in Sec. II a pattern of stripes undergoes a zigzag instability when a gradual increase in $\mu$ is applied (Fig. 1). This means that the stability bound for zigzags moves away from the line $q=0$ predicted by the phase equation (4). For patterns with negative excess wave numbers $q$, the zigzag instability leads to a fast waving of stripes, which ends in reconnections between stripes. This amplitude instability progresses differently depending on the value of $\mu$, as shown in Fig. 5. For low values of $\mu, H_{\pi}$ hexagons grow globally from stripes, while for high values of $\mu, H_{0}$ spots form locally at given places inside the pattern. These tran- 

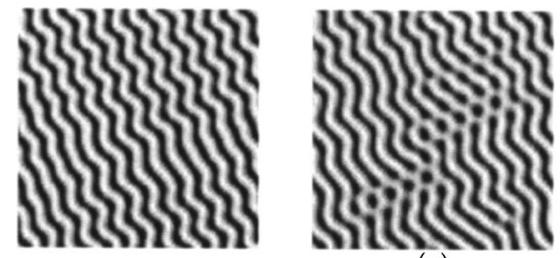

(a)
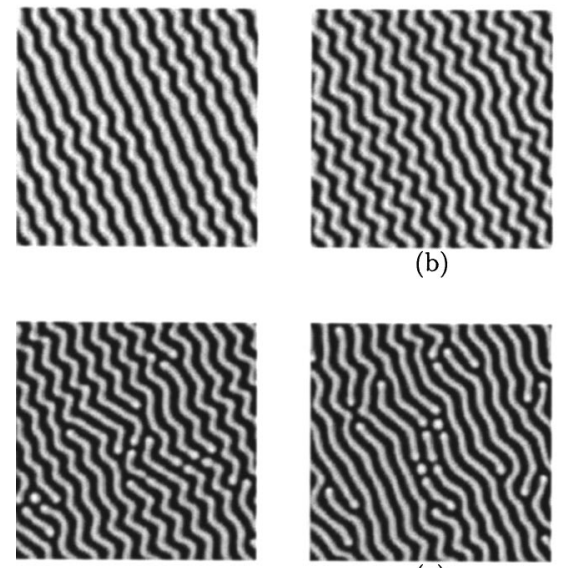

(b)
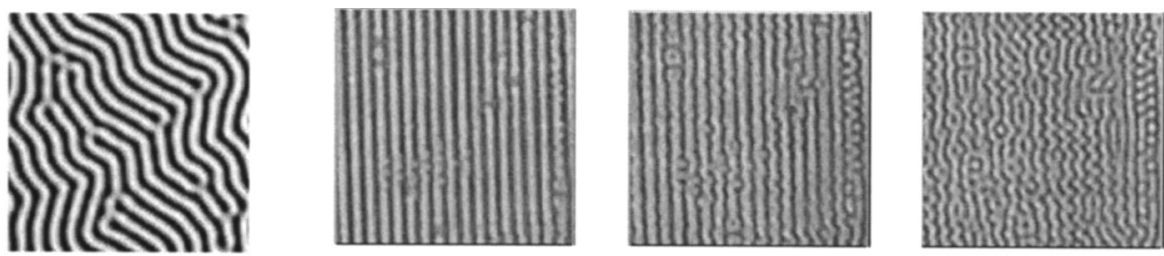

(a)
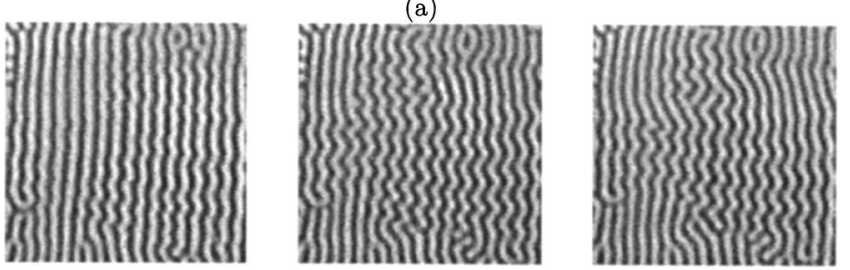

(b)

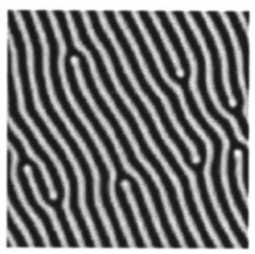

FIG. 5. Temporal evolution of the Brusselator with initial stripes unstable against the zigzag instability for (a) $\mu=0.10, q=-0.12$; (b) $\mu=0.15, q=-0.13$; (c) $\mu=0.65, q=-0.08$.

sient spots contribute to rearrange the wavy stripes which end up in straighter rolls with many point defects and with a wave number inside the stable band. At intermediate values of $\mu$, for which only stripes are stable, undulating rolls reconnect at some places leading to a kind of oblique grain boundary. These features are also in agreement with early experiments in Ref. [29].

\section{B. Experimental zigzag patterns}

The experimental sequence in Fig. 6(a) has been obtained by printing an initial pattern of stripes with $\lambda=0.62 \mathrm{~mm}$ $>\lambda_{c}=0.54 \mathrm{~mm}$. Snapshots in the upper row correspond to $\left[\mathrm{ClO}_{2}\right]=0.07 \mathrm{mM}$ (close to the region of stability of hexagons). The first snapshot in Fig. 6(a) shows the pattern after $15 \mathrm{~min}$. Here, hexagonal arrays of spots at the bottom and in a stripe to the right can be made out, and several defects distinguished. After $195 \mathrm{~min}$, the fine initial bands become rougher and slightly unfolded at some spots. In other places strong undulations are distinguished. The process continues with ruptures and reconnections between the stripes, in such a way that in the right pattern in Fig. 6 there is a stable mixture of zigzag patches with undulations, reconnections, and spots in a messy pattern. After $610 \mathrm{~min}$ the pattern is still very disordered, but the wave number has increased, again reaching the critical value $\lambda_{c}=0.54 \pm 0.02 \mathrm{~mm}$. Although this pattern differs substantially from the simulations in Figs. 5(a) and 5(c), the nucleation of spots seems crucial in readjusting the wave number.

Pictures in Figs. 6(b) show the evolution for $\left[\mathrm{ClO}_{2}\right]$ $=0.09 \mathrm{mM}$ (far from the hexagon-stripe transition) and the same wavelength as before. The initial stripes become wavy
FIG. 6. Experimental zigzag instability in CDIMA reaction for two cases. (a) $\left[\mathrm{ClO}_{2}\right]=0.07 \mathrm{mM}: t=0,195$, and $610 \mathrm{~min}$. (b) $\left[\mathrm{ClO}_{2}\right]=0.09 \mathrm{mM}: t=0,40$, and $180 \mathrm{~min}$. Both sequences show the temporal evolution of stripes with an initial wave number smaller than $\lambda_{c}$.

almost immediately. Undulations give rise to zigs and zags, apparent at the center picture in Fig. 6(b), after 40 min. Later on (at $t=180 \mathrm{~min}$ ), the pattern on the right in Fig. 6(b) is finally established. Notice that during this evolution hexagons did not play any role.

\section{CONCLUSIONS}

In this paper, we have investigated the sideband instabilities of Turing patterns of stripes in the Brusselator model and in the CDIMA reaction. Beginning with the amplitude equations for the main active modes in the pattern, we discussed under what conditions a long wavelength instability can be precluded by a transversal amplitude instability. Sufficiently close to the hexagon-stripe transition, this instability can give rise to distorted hexagons that evolve into a striped pattern with a stable wave number. The influence of the unstable hexagons should be much less far from the hexagon-stripe border.

In the Brusselator model, we studied numerically the stability regions of stripes, which differ substantially from the curves predicted within the amplitude equation framework. Experimentally, it has been confirmed that illumination permits the control of Turing patterns in the CDIMA reaction. By forcing an initial pattern with a wavelength different from the critical one, we observed an evolution that is similar to the dynamics reported in numerical simulations. The experimental control parameter is the concentration of chlorine dioxide $\left[\mathrm{ClO}_{2}\right]$. We considered two values of $\left[\mathrm{ClO}_{2}\right]$, one close $\left(\left[\mathrm{ClO}_{2}\right]=0.07 \mathrm{mM}\right)$ and another far from the hexagonstripe transition $\left(\left[\mathrm{ClO}_{2}\right]=0.09 \mathrm{mM}\right)$.

For too small wave numbers close to the stability ranges of hexagons, transient spotlike defects grow locally among stripes in numerical simulations and in experiments. The final pattern consists of stripes with a stable wave number. Far from the hexagon-stripe transition, the Eckhaus instability leads to the creation of defect pairs which subsequently annihilate each other, yielding a stable striped pattern with a 
slightly modified wave vector.

When initial stripes are too wide they display undulations and zigzags. Undulating stripes are found to be stable in a full range of slightly off-optimal wave numbers. If the impressed wavelength is increased, transient undulations give rise to domains of zigs and zags. Finally, if the initial wavelength is still larger, strong zigzags are reconnected at some places where amplitude defects are formed. Above but close to the stability region for hexagons, spots arise among the stripes. Far from the hexagon-stripe transition, however, the zigzag instability is not influenced by unstable hexagons, and disclinationlike defects and grain boundaries are observed.

We notice that, although the Brusselator model does not reflect the particular details of the CDIMA reaction, results of the modulational instabilities in simulations of this model and in experiments with the CFUR chemical reactor are in good qualitative agreement.

\section{ACKNOWLEDGMENTS}

The authors wish to thank J. Burguete (Pamplona) for helpful discussions. This work has been supported by the MCyT (Spanish Government) under Grants No. BFM2002O1002 (Pamplona) and No. BFM2000-0348 (Santiago), and by the PIUNA (Univ. Navarra). One of us (B.P.) acknowledges the financial support from the "Asociación de Amigos Univ. Navarra."
[1] A. Turing, Philos. Trans. R. Soc. London, Ser. B 237, 37 (1952).

[2] V. Castets, E. Dulos, J. Boissonade, and P. De Kepper, Phys. Rev. Lett. 64, 2953 (1990).

[3] I. Lengyel, G. Rabai, and I. R. Epstein, J. Am. Chem. Soc. 112, 9104 (1990).

[4] G. Gunaratne, Q. Ouyang, and H. L. Swinney, Phys. Rev. E 50, 2802 (1994).

[5] M. Waltz and A. F. Münster, Chem. Phys. Lett. 242, 273 (1995).

[6] F. Fecher, M. E. P. Strasser, F. W. Scheider, and A. F. Münster, Chem. Phys. Lett. 313, 205 (1999).

[7] M. Orbán, K. Kurin-Csörgei, A. M. Zhabotinsky, and I. R. Epstein, J. Phys. Chem. B 103, 36 (1999).

[8] O. Steinbock, E. Kasper, and S. C. Müller, J. Phys. Chem. A 103, 3442 (1999).

[9] Y.-J. Li, J. Oslonovitch, N. Mazouz, F. Plenge, K. Krischner, and G. Ertl, Science 291, 2395 (2001).

[10] M. Lowe and J. P. Gollub, Phys. Rev. Lett. 55, 2575 (1985).

[11] M. C. Cross and P. C. Hohenberg, Rev. Mod. Phys. 65, 851 (1993).

[12] V. Dufiet and J. Boissonade, Physica A 188, 158 (1992).

[13] A. P. Muñuzuri, M. Dolnik, A. M. Zhabotinsky, and I. R. Epstein, J. Am. Chem. Soc. 121, 8065 (1999).

[14] P. Manneville, Dissipative Structures and Weak Turbulence (Academic, San Diego, 1990).

[15] A. De Wit, Ph.D. thesis, Université Libre de Bruxelles, 1993.

[16] B. Peña and C. Pérez-García, Europhys. Lett. 51, 300 (2000).

[17] B. Peña and C. Pérez-García, Phys. Rev. E 64, 056213 (2001).
[18] P. Borckmans, A. D. Wit, and G. Dewel, Physica A 188, 137 (1992).

[19] B. Rudovics, E. Barillot, P. W. Davies, E. Dulos, J. Boissonade, and P. De Kepper, J. Phys. Chem. A 103, 1790 (1999).

[20] A. K. Horváth, M. Dolnik, A. P. Muñuzuri, A. M. Zhabotinsky, and I. Epstein, Phys. Rev. Lett. 83, 2950 (1999).

[21] A. Sanz-Anchelergues, A. M. Zhabotinsky, I. R. Epstein, and A. P. Munuzuri, Phys. Rev. E 63, 056124 (2001).

[22] W. Eckhaus, Studies in Nonlinear Stability Theory (SpringerVerlag, New York, 1965).

[23] E. Bodenschatz, M. Kaiser, L. Kramer, W. Pesch, A. Weber, and W. Zimmerman, in New Trends in Nonlinear Dynamics and Pattern-Forming Systems, edited by P. Coullet and P. Huerre (Pergamon, New York, 1990).

[24] The small parameter $\alpha$ for the Brusselator decreases when $\mu$ increases, so that it vanishes somewhere between the stability regions for hexagons $\left(H_{\pi}\right.$ and $\left.H_{0}\right)$. Around this particular value, the system is well described by the NWS equation because hexagons are not stable solutions. Consequently, no trace of hexagons should be seen under these circumstances, as confirmed in Fig. 3(b).

[25] S. Fauve, in Hydrodynamics and Nonlinear Instabilities, edited by C. Godrèche and P. Manneville (Cambridge University Press, Cambridge, U.K., 1998).

[26] K. Kawasaki and T. Ohta, Physica A 116, 573 (1982).

[27] A. C. Newell, T. Passot, N. Ercolani, and R. Indik, J. Phys. I 5, 1863 (1995).

[28] F. H. Busse and M. Auer, Phys. Rev. Lett. 72, 3178 (1994).

[29] Q. Ouyang and H. Swinney, in Chemical Waves and Patterns, edited by R. Kapral and K. Showalter (Kluwer, Dordrecht, Netherlands, 1995), p. 269. 